\title{
Structural Properties of Nanostructured Carbonate Apatites
}

\author{
C.A.O.Ramirez ${ }^{1, a}$, A.M.Costa ${ }^{1, b}$, J.Bettini ${ }^{2, c}$, A.J.Ramirez ${ }^{2, d}$, \\ M.H. Prado da Silva ${ }^{3, e}$ and A.M.Rossi ${ }^{1, f}$ \\ ${ }^{1}$ Labiomat/CBPF, Rua Dr. Xavier Sigaud 150, CEP 22290-180, Rio de Janeiro, Brazil \\ ${ }^{2}$ LME/LNLS, Rua Giuseppe Máximo Scolfaro 10000, CEP 13083-970, Campinas, Brazil \\ ${ }^{3}$ IME, Praça General Tibúrcio 80, CEP 22290-270, Rio de Janeiro, Brazil \\ acospina@cbpf.br, ${ }^{\mathrm{b}}$ andrea_machadocosta@yahoo.com.br, ${ }^{c}$ bettini@Inls.br, ${ }^{\mathrm{d}}$ ramirez@Inls.br, \\ émarceloprado@ime.eb.br rossi@cbpf.br
}

Keywords: Carbonate apatite, HRTEM.

\begin{abstract}
B-type carbonate apatite samples were synthesized by wet chemical method and characterized by X-ray Fluorescence Spectrometry, X-ray Diffraction, Fourier Transformed Infrared Spectroscopy and High Resolution Transmission Electron Microscopy. The XRD and FTIR analysis confirmed the presence of one B-type carbonate apatite phase and the HRTEM images revealed the coexistence of amorphous and polycrystalline regions in the order of $2 \mathrm{~nm}$ with the carbonate apatite structure. Second phases or precursors were not discovered.
\end{abstract}

\section{Introduction}

The inorganic phase of the calcified tissues is characterized as hydroxyapatite (HA), $\mathrm{Ca}_{10}\left(\mathrm{PO}_{4}\right)_{6}(\mathrm{OH})_{2}$, containing several ionic substitutions in the lattice sites occupied by the calcium, phosphate and hydroxyl ions [1]. The main substitution is the replacement of the hydroxyl (A sites) and the phosphate groups (B sites) by the carbonate ions [2]. These substitutions induce important modification on physical-chemical properties of HA structure and contribute to change HA biological response. The knowledge of the crystallization mechanisms of synthetic carbonated apatites could brings insights of the biological apatites structural features, as well as being fundamental to the development of new biomimetic materials for biomedical applications. However, few works have been made concerning the characterization of the carbonate apatites with nanosized crystals. In this work, HRTEM technique was used in order to characterize B-type carbonate apatite nanocrystals [3].

\section{Materials and Methods}

B-type carbonate apatites containing 5-7 wt $\% \mathrm{CO}_{3}$ were prepared by wet chemical method by the addition of aqueous solution of ammonium phosphate dibasic and ammonium carbonate on the calcium nitrate tetrahydrate at different temperatures (low and high temperature, labeled as CHA1 and $\mathrm{CHA} 2$, respectively) and $\mathrm{pH}=12$. Samples were collected at different aging times, filtrated and washed with deionized water. Sample characterization by electron microscopy using a HRTEMJEM 3010 URP microscope, was carried out at $300 \mathrm{kV}$, with point resolution of $0.17 \mathrm{~nm}$, spherical and chromatic aberrations of $0.6 \mathrm{~mm}$ and $1.2 \mathrm{~mm}$, respectively, Scherzer defocus of $-42 \mathrm{~nm}$ and energy spread of $1.6 \mathrm{eV}$. The mineral phases presented on the apatite samples were analyzed by powder X-ray diffraction using a PANalytical X'Pert PRO X-Ray Diffractometer, operating at $40 \mathrm{kV}$, with $\mathrm{CuK}_{\alpha 1}$ radiation and $40 \mathrm{~mA}$. The vibrational modes were obtained by the use of the IRPrestige-21 series Fourier Transform Infrared Spectrophotometer in the range of $4000-400 \mathrm{~cm}^{-1}$, with 64 scans, resolution of $4 \mathrm{~cm}^{-1}$. Chemical analysis was carried out with the PW2400 XRF Spectrometer, operating at $40 \mathrm{kV}, 50 \mathrm{~mA}$ and using a germanium crystal for phosphorus and calcium lines determination $(24 \mathrm{kV}, 70 \mathrm{~mA}$ and PX4 crystal for carbon lines determination). 


\section{Results and Discussion}

$\mathrm{XRF}$ analysis showed that $\mathrm{Ca} / \mathrm{P}$ ratio varied from 1.68 to 1.70 and the carbonate content between $5 w t \%$ and $7 w t \%$. XRD and FTIR characterization (Fig. 1 and Fig. 2, respectively) confirmed that samples were constituted of carbonated hydroxyapatite with an amorphous structure in the early stages of the crystallization process. Samples that were precipitated at $90^{\circ} \mathrm{C}$ (CHA2) showed a typical XRD pattern of a crystalline hydroxyapatite as shown in Fig.1a. Precipitation at low temperature produced non ordered structures, as seen in Fig. 1b The FTIR spectrum of sample synthesized at high temperature showed the typical bands of hydroxyapatite. The presence of the carbonate bands at $870 \mathrm{~cm}^{-1}$ and at the $1400-1500 \mathrm{~cm}^{-1}$ region confirms the $\mathrm{A}\left(\mathrm{OH}^{-}\right)$and $\mathrm{B}\left(\mathrm{PO}_{4}{ }^{3-}\right)$ substitutions; the former in very small concentrations. The broad FTIR bands of sample CHA1 indicated that carbonated HA precipitated at low temperature had a disordered structure probably due to the large amount of water molecules which were incorporated into the HA lattice.

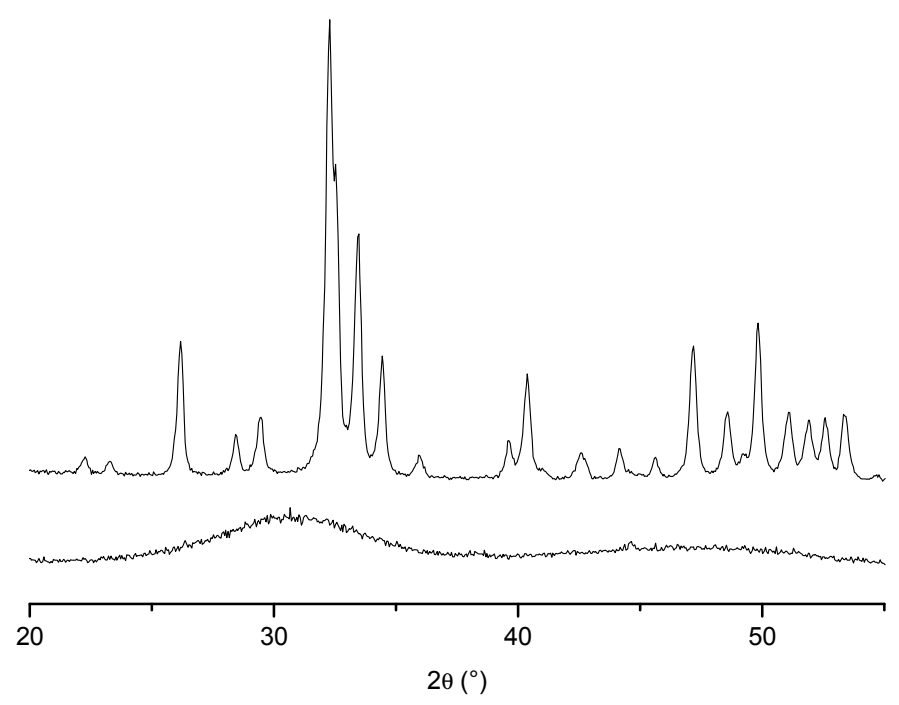

Fig.1 XRD patterns of the CHA1 and CHA2 samples (from bottom to the top).

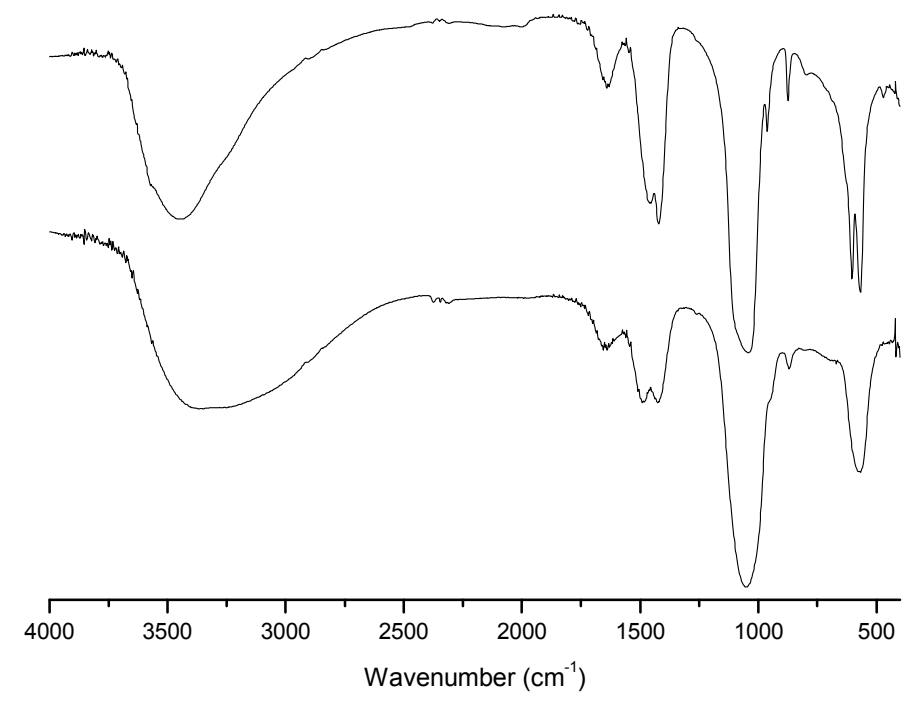

Fig.2 FTIR spectra of the CHA1 and CHA2 samples (from bottom to the top). 
Conventional transmission electron microscopy images (not showed) showed that carbonated apatite powder is formed by large agglomerates of nanoparticles with irregular shape. In general, agglomerate size was about $150 \mathrm{~nm}$, while individual particles were smaller than $30 \mathrm{~nm}$. In nanometric scale, the high resolution images (Fig. 3) revealed the coexistence of amorphous and crystalline regions, associated to a carbonate apatite structure. The sample precipitated at $90^{\circ} \mathrm{C}$ presented well defined crystalline edges, corresponding to the (110) cleavage plane and a superposition of crystal domains in the order of $5 \mathrm{~nm}$ or less. Samples synthesized at lower temperature and with 0 hours of aging time, were composed by crystalline nanoparticles (Fig. 3b), which amorphized under electron beam interaction. In some cases samples with 2 hours of aging time (Fig. 3c) were initially amorphous and then crystallized by the prolonged electron beam interaction. The Energy-dispersive X-ray spectroscopy showed the peaks of $\mathrm{Ca}, \mathrm{P}, \mathrm{O}$ and $\mathrm{C}$ elements and the $\mathrm{Ca} / \mathrm{P}$ ratios were quite different from 1.67 , due to the carbonate substitution and by P sputtering [4].

The Fast Fourier Transformed (FFT) of the CHA2 images using the DigitalMicrograph software confirmed the stretching of hydroxyapatite $c$-axis caused by the incorporation of the carbonate on phosphate site [5]. Hydroxyapatite reflection planes along [513], [210], [421] zone axes are observed in FFT images of CHA1 and CHA2. HRTEM images simulations showed good agreements with the experimental images (result not shown). Reflection planes from possible precursors of hydroxyapatite or additional mineral phases were not found.
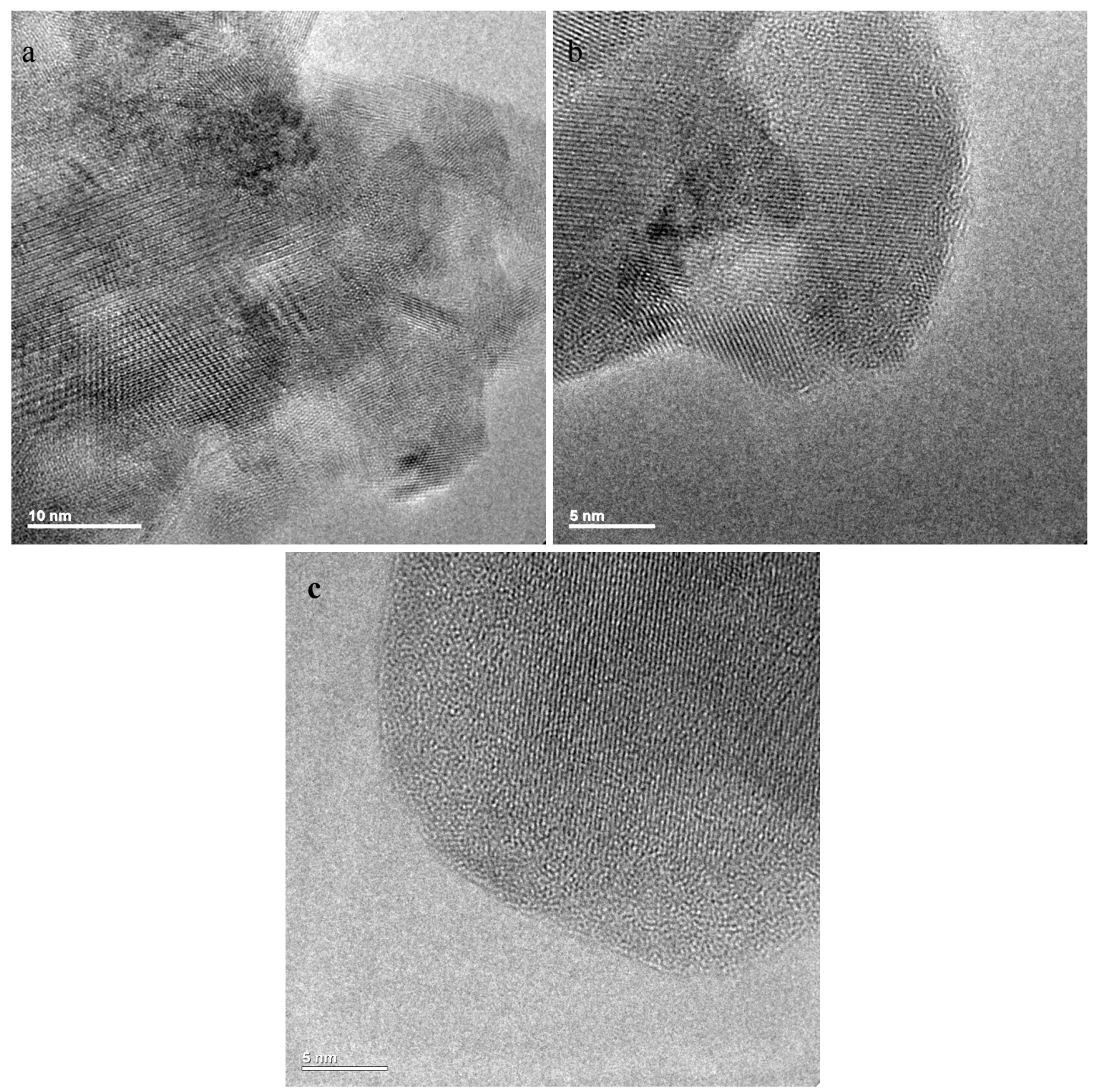

Fig.3 HRTEM images of CHA2 (a), CHA1 samples with 0 hour (b) and 2 hours of aging time (c). 


\section{Conclusions}

Carbonate apatite particles precipitated at temperature lower than $25{ }^{\circ} \mathrm{C}$ had a predominantly amorphous character. Carbonate ions were incorporated in both phosphate and hydroxyl sites. HRTEM analysis suggested that the formation of the B-type carbonate apatite is not preceded by a precursor. The crystallization model proposed in the literature based in the hypothesis that particles were made of a crystalline core and an outer amorphous layer was not confirmed by this study.

This work received the financial support of the Brazilian agency CNPq and the electron microscopy analysis was carried out on the LME/LNLS/Campinas.

\section{References}

[1] R. Murugan and S. Ramakrishna: Composites Science and Technology 65, Issues 15-16 (2005), p. 2385.

[2] R. Z. LeGeros, in: Calcium Phosphates in Oral Biology and Medicine. Monographs in Oral Sciences, Vol. 15, Karger (1991).

[3] A.M. Rossi, M.H. Prado da Silva, A.J. Ramirez, D. Biggemann, M.M. Caraballo, Y.P. Mascarenhas, J.G. Eon and G.T. Moure: Key Engineering Materials Vols.330-332 (2007), p. 255.

[4] D. Biggemann et al: Microscopy and Microanalysis (2008), submitted.

[5] R.M. Wilson, Stephanie E.P. Dowker and J.C. Elliott: Biomaterials 27 (2006), p.4682. 